\title{
PERIPARTUM CARDIOMYOPATHY: A CASE REPORT
}

\author{
R. Sowjanya ${ }^{1}$, K. Saritha ${ }^{2}$, T. Shravya ${ }^{3}$
}

\section{HOW TO CITE THIS ARTICLE:}

R. Sowjanya, K. Saritha, T. Shravya. "Peripartum Cardiomyopathy: A Case Report". Journal of Evolution of Medical and Dental Sciences 2015; Vol. 4, Issue 27, April 02; Page: 4731-4736, DOI: 10.14260/jemds/2015/686

ABSTRACT: Peripartum cardiomyopathy (PPCM) is a rare form of heart failure with a reported Incidence of 1 per3000 to 1 per 4000 live births and a fatality rate of $20-50 \%$.Onset is usually between the last month of pregnancy and up to 5 months postpartum in previously healthy women. Although viral autoimmune and idiopathic factors may be contributory, its etiology remains unknown. PPCM is usually presents with signs and symptoms of congestive heart failure. Early diagnosis is important and effective treatment reduces mortality rates and increases the chance of complete recovery of ventricular systolic function.

KEYWORDS: Peripartum cardiomyopathy, pregnancy, heart failure, echocardiography.

INTRODUCTION: Peripartum cardiomyopathy (PPCM) is a rare form of heart failure of unknown cause occurring between the last month of pregnancy and up to 5 months postpartum in previously healthy women. ${ }^{1}$ Symptoms of PPCM, which include fatigue, oedema and dyspnoea, are similar to those for the normal spectrum of peripartum states and pregnancy comorbidities, such as pulmonary emboli and eclampsia. Therefore, diagnosis is often delayed and the disorder is under recognized, with devastating consequences. Mortality is as high as $20-50 \% .^{2}$ We report an interesting case of peripartum cardiomyopathy and discuss about the clinical presentation, therapy and outcome of this condition.

CASE REPORT: A 22 year old $\mathrm{G}_{2} \mathrm{P}_{1} \mathrm{~L}_{1}$ with previous history of Caesarean section who was booked in our antenatal OP and was decided for a vaginal birth after caesarean (VBAC) was admitted with history of labour pains one day prior to her expected date of delivery. The patient collapsed one hour after admission, complaining of sudden onset of breathlessness and chest pain. On examination, patient was irritable but conscious, pulse rate 112 per minute, respiratory rate 28 breaths per minute, blood pressure was $80 / 50 \mathrm{~mm} \mathrm{Hg}$ and extremities were cold and clammy. On auscultation heart and lungs were normal. Patient was resuscitated with oxygen and vasoactive drugs (Noradrenaline and dopamine).

Per abdomen uterus was term, acting mildly and foetal heart sounds were present and tachycardia was present. Per vaginal examination revealed cervix was $50 \%$ effaced and os dilated to $1 \mathrm{Cm}$, the presenting part was at ' 0 ' station and pelvis was gynaecoid and adequate.

Her ECG showed sinus tachycardia, Chest X- ray revealed massive cardiomegaly with haziness at right base of lung. As her restlessness increased and her oxygen saturation started dropping inspite of oxygen administration and resuscitative measures, the patient was intubated and connected to ventilator. Emergency 2D ECHO revealed global hypokinesia, severe LV systolic dysfunction, ejection fraction 25-30\%, No tricuspid or mitral regurgitation, no clot. Diagnosis of peripartum cardiomyopathy was made. Treatment was modified as per cardiologist advice and dobutamine infusion was started. 


\section{CASE REPORT}

Meanwhile foetal bradycardia was noticed and emergency LSCS was planned but patient's relatives did not give consent for LSCS. 12 hours after admission while still on ventilator the patient delivered a live female baby weighing $2.8 \mathrm{~kg}$ by outlet forceps. Baby's Apgar was 4. Baby was resuscitated and shifted to neonatal ICU.

After delivery the patient was transferred to Cardiology unit for further management. With treatment patient showed signs of improvement, severe LV dysfunction improved to mild LV dysfunction with ejection fraction of $45 \%$ after three weeks.

DISCUSSION: The peripartum cardiomyopathy (PPCM) is classified as a specific cardiomyopathy that develops in the last period of pregnancy or in the first period Puerperium.3,4

PPCM is defined on the basis of the following criteria, adapted from Demakis et al.5,6:

- Development of cardiac failure in the last month of pregnancy or within 5 months of delivery;

- Absence of recognizable heart disease prior to the last month of. pregnancy;

- Absence of identifiable cause for the cardiac failure;

- Left ventricular systolic dysfunction demonstrated by classic echocardiographic criteria, such as depressed shortening fraction or ejection fraction.

ETIOLOGY: The cause of PPCM is still unknown. Most postulate that it is related to the maladaptive response to haemodynamic stress of pregnancy (Increased fluid load). During pregnancy there are some alterations in the haemodynamic set-up, ${ }^{7}$ with subsequent transient hypertrophy. ${ }^{8}$ In the second and third trimesters of pregnancy a reversible decrease in the left ventricular systolic function occurs, it persists up to the early postpartum period, but returns to baseline thereafter. It is possible that PPCM is due to exaggeration of this decrease in the systolic function. ${ }^{9}$

Other researchers further postulate that PPCM may be an inflammatory response in pregnancy, citing an elevation of tumour necrosis factor-alpha and interleukin-6 levels. ${ }^{10,11}$

Another important factor, which may cause PPCM, is the abnormal immune response to pregnancy associated with high titres of autoantibodies against particular cardiac proteins. ${ }^{12}$ Randet al ${ }^{13}$ postulated an immunological cause on the basis of the presence of heart muscle antibodies in cord blood and serum of neonates born from cardiomyopathic mother. The authors show that, after delivery, the fast degeneration of the uterus results in fragmentation of tropocollagen by collagenolytic enzymes releasing actin, myosin, and their metabolites; these antibodies are formed against actin and cross-react with the myocardium. It has also been suggested that PPCM may have viral cause. ${ }^{14}$ In the light of the presence of dense lymphocytic infiltrate, myocyte oedema, necrosis and fibrosis in ventricular biopsies of patients with PPCM, Melvin et al ${ }^{15}$ proposed myocarditis as the cause of PPCM. This hypothesis is consistent with the clinical improvement that is typically associated with immunosuppressive treatment (Prednisolone and azathioprine)

Multiparity, advanced maternal age, multifoetal pregnancy, pre-eclampsia, gestational hypertension and African ethnicity are risk factors. Other risk factors include association with maternal cocaine abuse or selenium deficiency, long term (more than four weeks), oral tocolytic therapy with beta adrenergic agonists such as terbutaline. However, the disease can occur in women without any risk factors. ${ }^{1}$

Some familial cases of PPCM have been reported, raising the possibility that sometimes PPCM is actually a familial dilated cardiomyopathy unmasked by the pregnancy. ${ }^{16}$ 
Other possible etiological factors include excessive ingestion of salt. Abnormalities of relaxin, an ovarian hormone produced during pregnancy, can have positive ionotropic and chronotropic properties and cause excessive relaxation of the cardiac skeleton. Deficiency of selenium may increase the heart susceptibility to viral infection, hypertension or hypocalcaemia. It is unclear whether nutritional deficiencies may play a role in the pathogenesis of PPCM. ${ }^{14}$

Clinical Features: Clinical features of PPCM include symptoms of congestive heart failure and chest pain. Signs can include tachycardia, tachypnoea, pulmonary rales, an enlarged heart, and third heart sound. Such signs and symptoms overlap with those of many other conditions, ranging from normal pregnancy to pulmonary emboli and upper respiratory infection. ${ }^{1}$ Diagnosis of PPCM includes the four criteria described by Demakis et al.

Symptoms like decreased exercise capacity, tiredness, dyspnoea, orthopnoea and palpitations may occur even in normal pregnancy and can be mistaken for a diseased state. Physical signs like hyperventilation, oedema and distended neck veins can be seen in normal pregnancy. On auscultation of the heart, one can hear loud first heart sound, exaggerated splitting, mid systolic murmur and continuous venous hum. These physical signs may confuse the clinician and there could be mistakes in the form of over diagnosis or disregarding of heart disease.17,18 The clinical presentation and haemodynamic features in PPCM are indistinguishable from those of other forms of dilated cardiomyopathy.

Investigations: There are no specific laboratory abnormalities for PPCM. However, other exclusionary laboratory studies should also be considered, including cardiac enzymes assessment and a pre-eclampsia workup.

Chest radiographs may show cardiomegaly with pulmonary oedema and pulmonary venous congestion, and pleural effusions in some cases. The electrocardiogram may show sinus tachycardia, nonspecific ST and T wave changes, atrial or ventricular arrhythmias and conduction defects. Echocardiographic/ Doppler examination may reveal enlargement of all four chambers with marked reduction in left ventricular systolic function, small to moderate pericardial effusion and mitral, tricuspid and pulmonary regurgitation may be evident. Ventricular wall motion, ejection fraction and cardiac output are decreased and pulmonary wedge pressure is increased.19,20,21

Differential Diagnosis ${ }^{14:}$ The diagnosis of PPCM should be seriously considered in all patients with persistent or worsening heart failure in the last month of pregnancy or in the early puerperium. When the diagnosis of PPCM is considered, nearly every other cause of left ventricular dysfunction must be excluded such as myocardial infarction, sepsis, severe pre-eclampsia, pulmonary embolism, idiopathic dilated cardiomyopathy, valve disease ( mitral and aortic stenosis ) and pulmonary vasculitides (Systemic lupus erythematosus, scleroderma, rheumatoid disease) Idiopathic dilated cardiomyopathy has clinical characteristics similar to PPCM, but the onset is not restricted to the peripartum period can occur in the second trimester

Treatment: 2D ECHO is valuable in diagnosing PPCM, formulating prognosis for recovery and following course of disease, especially EF and LV end diastolic dimensions are predictive of long term cardiac dysfunction. 
The treatment for PPCM is the same as for other forms of congestive heart failure (fluid and salt restriction, $\beta$-blocker, diuretic, and digoxin), except for angiotensin converting enzyme inhibitors and angiotensin-receptor blockers, which are contraindicated in pregnancy. ${ }^{22}$ Hydralazine can be used during pregnancy to reduce after load. Patients with PPCM are also at high risk for thrombus formation; thus, anticoagulation should be considered especially for high-risk patients with severe LV dysfunction. In addition, physical activity should be encouraged according to patients' tolerance of symptoms. Immunosuppressive therapy has no clear cut efficacy and, therefore, is not currently recommended. Women who fail maximal medical management may be candidates for cardiac transplantation. ${ }^{1}$

It has been hypothesized that early blockade of prolactin with bromocriptine may improve the condition of patients with acute onset of PPCM before irreversible damage occurs. The beneficial effect results from eliminating the detrimental $16 \mathrm{kDa}$ prolactin form. Bromocriptine was also shown to increase oxygen consumption and resting energy expenditure. Anticoagulation therapy is strongly recommended as this has been associated with several reports of myocardial infarction. ${ }^{23}$

Prognosis: The clinical course of PPCM varies with approximately 50-60\% patients showing complete or near complete recovering of clinical status and cardiac function, usually within the first 6 months postpartum. The remaining patients demonstrate either continued clinical deterioration leading to early death or persistent left ventricular dysfunction and chronic heart failure. There appears to be an initial high risk period with a mortality of $25-50 \%$ in the first 3 months postpartum. Patients with persistent cardiomegaly at 6 months have a reported mortality of $85 \%$ at 5 years. . $^{20,24,25}$

The persistence of cardiac dysfunction 6 to 12 months after the initial diagnosis of PPCM usually indicates an irreversible problem and almost always represents an absolute contraindication to a subsequent pregnancy. The data have been conflicting in patients with PPCM in whom left ventricular function recovers. But these women appear to have some risk for recurrence. Regardless of recovery, however, a second pregnancy is usually not recommended for these patients because PPCM recurs in more than $30 \%$ of subsequent pregnancies, which puts both mother and baby at great risk. ${ }^{1}$

CONCLUSION: PPCM is a rare disease, the cause of which is unknown, typical of women of childbearing age. Diagnosis is difficult and requires vigilance. Prompt diagnosis and initiation of appropriate medical management in collaboration with cardiologist is essential to provide the best possible outcome for both mother and baby.

\section{REFERENCES:}

1. Sreelatha S, Shilpashree M, Ashwini Rani M, Ramaiah R. Peripartum cardiomyopathy - A case report. Med Inn 2013; 2: 120-22.

2. Abboud J, Murad Y, Chen-Scarabelli C, Saravolatz L, Scarabelli TM. Peripartum cardiomyopathy: a comprehensive review. Int J Cardiol 2007; 118 (3): 295-30.

3. Homans DC. Peripartum cardiomyopathy. N Engl J Med 1985; 312: 1432-7.

4. Lampert MB, Lang RM Peripartum cardiomyopathy. Am Heart J 1995; 130: 860-70.

5. Demakis JG, Rahimtoola SH. Peripartum cardiomyopathy. Circulation 1971; 44 (5): 964-8. 


\section{CASE REPORT}

6. Demakis JG, Rahimtoola SH, Sutton GC, et al. Natural course of peripartum cardiomyopathy. Circulation 1971; 44 (6):1053-61.

7. Geva T, Mauer MB, Striker L, Krishon B, Pivamik JM. Effects of physiologic load of pregnancy on left ventricular contractility and remodelling. Am Heart J 1997; 133: 53-59.

8. Mone SM, Sanders SP, Colan SD. Control mechanism for physiological hypertrophy of pregnancy. Circulation 1996; 94: 667-672.

9. Julian DG, Szekeley P. Peripartum cardiomyopathy. Prog Cardiovasc Dis 1985; 27: 233-246.

10. Sliwa K, Skudicky D, Bergemann A, Candy G, Puren A Sareli P. Peripartum cardiomyopathy: analysis of clinical outcome, left ventricular function, plasma levels of cytokines and Fas/APO-I J Am Coll Cardiol 2000; 35 (3): 701-5.

11. Sliwa K, Förster 0, Libhaber E, et al. Peripartum cardiomyopathy: inflammatory markers as. predictors of outcome in 100 prospectively studied patients Eur Heart J 2006; 27(4):411-6.

12. Witlin AG, Mable WC, Sibai BM. Peripartum cardiomyopathy: an ominous diagnosis. Am J Obstet Gynecol 1997; 176:182-8.

13. Rand RJ, Jenkins DM, Scott DG. Maternal cardiomyopathy of pregnancy causing still birth. BJOG $1975 ; 82$.

14. Colombo BM and Ferrero S. Peripartum cardiomyopathy. Orphanet encyclopedia October2004.

15. Melvin KR, Richadson PJ, Olsen EG, Daly K, Jackson G. Peripartum cardiomyopathy due to myocarditis. N Engl J Med 1982; 307:731-4.

16. Pierce JA, Price BO, Joyce JW. Familial occurrence of postpartal heart failure. Arch Inter Med 1963; 111:651-5.

17. Uri Elkayam. Pregnancy and cardiovascular diseases. In: Braunwald, Zipes, Libby, editors. A text book of cardiovascular medicine. 7thedition. Saunders 2005: 1973-74.

18. Joshua Wynne. Eugene Braunwald. Cardiomyopathy and myocarditis. In: Kasper, Braunwald, Fauci et al editors. Harrison's Principles of Internal medicine. 16th edition, Mc Graw Hill 2005: 1408-14.

19. Vinod Sharma. Peripartum Cardiomyopathy. Cardiology Today; May-June 2004; 8(3): 127-29.

20. Carvallo A, Brandao A, et al. Prognosis in peripartum cardiomyopathy. Am J Cardiol; 1989; 64; 540-42.

21. Midei Mg et al. Peripartum myocarditis and cardiomyopathy, Circulation 1990; 81: 922-28.

22. Libby P, Bonow RO, Mann DL, Zipes DP, eds. Braun Wald's heart disease: a textbook of cardiovascular medicine. 8th ed. Philadelphia, PA: Saunders; 2007.

23. Hopp L, Harder B, Iffy L. Myocardial Infarction postpartum in patients taking bromocriptine for the prevention of breast engorgement. Int J cardiol 1996; 57: 227-32.

24. Michael de swiet. Heart disease in pregnancy. In: Michael de swiet. Editor. Medical disorders in obstetric practice. $4^{\text {th }}$ edition, Blackwell Scientific Publication 2002; 144-46.

25. F Garry Cunningham, Kenneth J Leveno, Steven L Bloom et al. Cardiovascular diseases. In: Dwight Rause, Bill Rainey, Cathy Spong, George Vendel Jr, editors. Williams Obstetrics. 22nd edition. Mc Graw Hill 2005: 1030-32. 


\section{CASE REPORT}

\section{AUTHORS:}

1. R. Sowjanya

2. K. Saritha

3. T. Shravya

\section{PARTICULARS OF CONTRIBUTORS:}

1. Assistant Professor, Department of Obstetrics and Gynaecology, Siddhartha Medical College, Vijayawada, Andhra Pradesh.

2. Associate Professor, Department of Obstetrics and Gynaecology, Siddhartha Medical College, Vijayawada, Andhra Pradesh.

3. Post Graduate, Department of Obstetrics and Gynaecology, Siddhartha Medical College, Vijayawada, Andhra Pradesh.

\section{NAME ADDRESS EMAIL ID OF THE CORRESPONDING AUTHOR:}

Dr. K. Saritha, Plot No. 15, Indian Airlines Colony, Opposite Football Grounds, Trimulgherry, Secunderabad-500015.

E-mail: saritha.kottu@gmail.com

Date of Submission: 09/03/2015. Date of Peer Review: 10/03/2015. Date of Acceptance: 20/03/2015. Date of Publishing: 02/04/2015.

FINANCIAL OR OTHER

COMPETING INTERESTS: None 